\title{
Research on vehicle monitoring system
}

\author{
Li Lun-bin ${ }^{a}$, Teng Hai-kun and Wang Li-hong \\ Heihe University, 164300, China
}

Keywords: Vehicle monitoring system, communication network, GIS, safety.

\begin{abstract}
The vehicle monitoring system is to use GPS technology to locate the vehicle, the vehicle information through the communication network back to the monitoring center, monitoring center and then use GIS technology to analyse the vehicle information, the vehicle is displayed on the electronic map, and the vehicle control. Study of vehicle monitoring system, so that the function is more perfect, to a large extent, improve the safety of vehicle operation and road utilization, has broad application prospects.
\end{abstract}

\section{Background and significance}

With the rapid development of economy, China's transportation industry is also developing rapidly, the number of vehicles every year is growing at an alarming rate. However, with the rapid increase of vehicles, vehicle monitoring technology and management technology has lagged behind, has brought many serious problems, such as vehicle theft, traffic accidents, traffic congestion, waste of resources, the driver's personal safety, environmental pollution, etc.. In order to adapt to the traffic of the rapid development, improve the urban traffic conditions, improving social security, ease traffic pressure, crack down on all kinds of grab the car, car theft activities and effective real-time management and monitoring of vehicles is very important, there is an urgent need to establish a fast and efficient, intelligent, real-time, command is in favour of the intelligent transportation system[1]. Vehicle monitoring field has great application value and market prospect, which is an important part of Transport System Intelligent (Intelligent Transportation System). Intelligent transportation system (ITS) is the advanced communication technology, information technology, control technology, computer technology and sensor technology, and effectively integrated into the entire transport management system, and the establishment of a global, all-weather, high performance, real time, efficient, accurate transportation and management system. It effectively improve road traffic capacity, ease road congestion and less traffic accidents, reducing the rob car theft event occurs, reduce environmental pollution, reduce the consumption of energy[2].

Vehicle monitoring system is a very important direction of ITS research. Vehicle monitoring system can provide a lot of important information, such as precise position of vehicle, vehicle direction, vehicle speed, vehicle driving speed, etc.. Vehicle monitoring center can be monitored by vehicle monitoring system to monitor the vehicle's situation as well as their driving situation, and when necessary, can give it to provide rescue information, such as vehicles in the vicinity of the hospital, etc.. Vehicle monitoring system can provide global, all-weather and high precision

\footnotetext{
${ }^{\mathrm{a}}$ Corresponding author : 763203449@qq.com
} 
navigation and positioning, can effectively improve the road utilization, ease traffic congestion[3]. This shows that the research and development of vehicle monitoring system has very important significance, which can provide us with high accuracy of real-time positioning information and intelligent navigation information, to ensure safe driving, improve road capacity, ease traffic congestion, but also reduce environmental pollution, save energy.

\section{Research statuses at home and abroad}

Intelligent transportation system is based on the vehicle monitoring system. Intelligent transportation system is a comprehensive application of advanced GPS global positioning technology, GIS electronic map technology, wireless communication technology, control technology and computer technology, etc.. In recent years, domestic and foreign intelligent transportation has become a hot spot, many countries have studied and achieved good results.

\subsection{The status quo of the development of foreign countries}

Traffic congestion, road congestion, grab a car theft, environmental pollution has become a very serious social problem, in recent years, with the rapid increase of vehicles has traffic to bring a lot of pressure [4]. So, how to effectively solve these problems? Since 1980s, in order to reduce the traffic pressure, increase the effective utilization rate of roads, enhance the safety, protect environment and save energy, developed countries, such as Canada, Japan, the United States and Germany, developed the intelligent transportation system to reduce the traffic pressure, reduce the traffic congestion, enhance the safety factor, enhance the traffic capacity, protect the environment and save energy. The main approach is to use the global positioning technology, electronic map technology, wireless communication technology and control technology to the electronic map, in order to manage, such as providing the driver with the location, the direction of the vehicle, the vehicle speed, the surrounding environment, to provide effective help, can effectively increase the capacity of the road, changed the traditional transportation. At present, the research of intelligent transportation system is studied in the aspects of vehicle safety and control, traffic model, traffic control and management, communication and broadcasting system. Vehicle positioning system and vehicle navigation system is the most widely used in intelligent transportation system [5].

\subsection{The status quo of domestic development}

Research in the field of intelligent transportation in our country began in 1980s; the government departments attach great importance to the research and development of intelligent transportation, Ministry of science and technology, Ministry of information industry and related universities and other scientific research institutions to research and develop intelligent transportation system, and have been included in China's high-tech development plan[6]. Starting in 1994, some companies began to design mobile information system, after two years, the company's research vehicle monitoring system has reached hundreds of companies, but the technology is relatively backward, many products are not pushed to the market, not put into use; starting in 2000, the vehicle monitoring system has become a hot market, many companies continue to produce more mature products, but the current use of technology is still very backward. In 1990s, we began to study and construct the mobile target monitoring system. With the development of various technologies, such as the improvement of global positioning technology, the development of electronic map technology, communication technology, software and hardware, and so on, it has promoted the development of mobile target monitoring system. At present, there are many monitoring system and navigation system, such as the digital map monitoring system and the digital map navigation system of the major cities. This shows that the major cities have begun to study and develop the intelligent transportation system, which indicates that the intelligent transportation system is a very good development prospect. But our 
country's information level is still low, the technology level is still relatively low, at present the work still focuses on the foundation of the intelligent transportation system[7].

\section{Technologies related to vehicle monitoring system}

\subsection{Global positioning systems}

Global positioning system is a wireless navigation system, it is the use of 24 satellites to locate. It can provide us with global, all-weather; high precision positioning, such as vehicle location, speed and direction, etc., at present, GPS global positioning system has become one of the main methods of vehicle monitoring. The basic principle of GPS positioning is to measure the distance between a user's receiver to a satellite, and then according to the satellite data, the receiver can be derived. The distance between the satellite and the user can be obtained by the time of the recording of the satellite signal. But the interference is not the real distance. The satellite clock recorded time to detect satellite location in satellite ephemeris. The GPS satellite will continue to consist of 1 and 0 binary symbols of pseudo random code transmitting navigation message. From the satellite signal solution modulation navigation message including satellite ephemeris and clock correction, working conditions, ionospheres' delay correction, atmospheric refraction correction etc. information and ephemeris data is navigation message is one of the most important content. The role of the satellite part of the GPS navigation system is to launch the navigation message. GPS receiver can receive the exact time information; used to calculate the required satellite coordinates of the broadcast ephemeris; for forecasting several months' satellite about location forecasting ephemeris and GPS system information and so on.

\subsection{Geographic information system technologies}

GIS technology is based on surveying and mapping, data stored in the database, the use of data from the database, to computer programming as a platform for global spatial analysis of instant technology. This is the core of GIS. The role of GIS in the field of vehicle monitoring is increasingly important. Monitoring center receives the information from the vehicle. The data is displayed on the electronic map to realize the monitoring of the vehicle, such as the speed, direction and position of the vehicle. In the vehicle monitoring system, the main functions of GIS are as follows: users can conveniently in the electronic map, such as zoom in, zoom out, map, map roam; to provide a visual interface, in the electronic map monitoring of vehicles, including the location of the vehicle, driving speed, driving direction and the vehicle driving around the environment, and so on; the user can query a vehicle in the electronic map.

\subsection{Map matching algorithm}

The monitoring center receives information from the GPS user terminal, including the speed, direction and location of the vehicle. The corresponding information is displayed on the electronic map. The electronic map is used to display the actual driving trajectory, including turning and driving section. This process is error, which is the most important problem in the vehicle monitoring system. Map matching is a method that the GPS vehicle monitoring system is modified. It is the path of the vehicle track that is obtained by GPS data information and digital map database module. It can be used to connect the vehicle position and road network. Map matching is a kind of software technology .

\section{Design and implementation of vehicle monitoring system}

Vehicle monitoring system is composed of four modules. The main function of vehicle tracking function: tracking, a vehicle tracking and display the vehicle real-time status, query function: 
according to the conditions of the query for a given vehicle and location on the map, map basic functions: the map of the basic operation, such as zoom in on the map, reduce the map and map display and so on, historical track playback function: replay a vehicle in a time interval of historical trajectory. Vehicle monitoring system function module diagram is shown in figure 1.

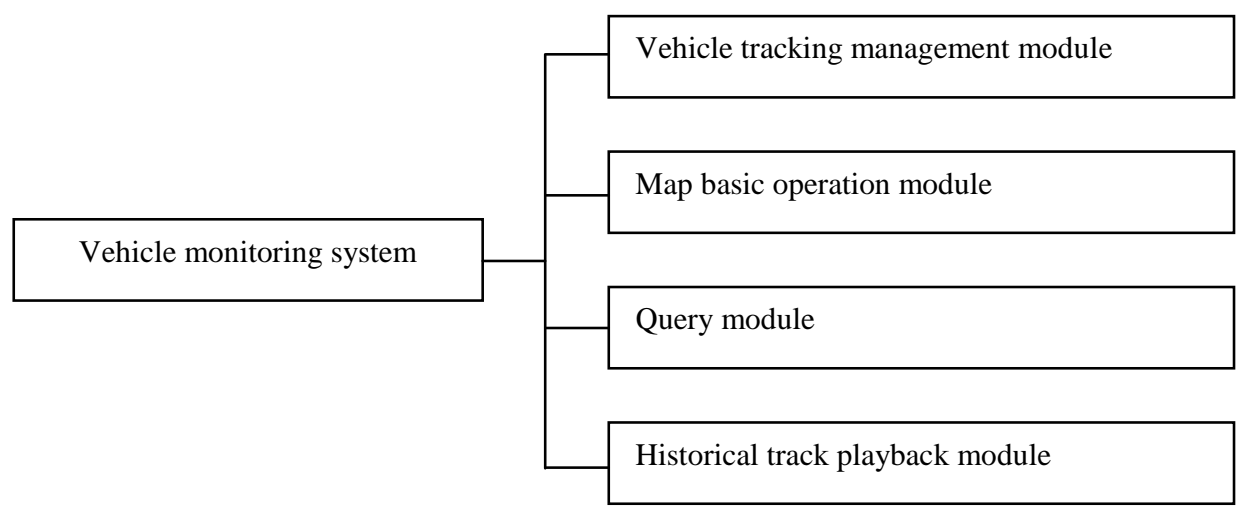

Figure 1. Vehicle monitoring system module function diagram

\subsection{The function of the vehicle tracking management module}

Real time display of vehicle location, which can obtain the vehicle's current position, and display the real-time position of the vehicle, the vehicle can be used to display the real-time status of the vehicle, which can be used to track the vehicle's function, which can be used to read the electronic map database.

\subsection{The function of the basic operation module of the map}

Out the map function, namely the vehicle monitoring system can be reduced to a range of electronic map; enlarge the map function, namely the vehicle monitoring system can enlarge a range on the electronic map; map map display function, namely the vehicle monitoring system can display the panoramic map; shift map function, namely the vehicle the monitoring subsystem can move the operation on the display of map display function; Hawkeye monitoring subsystem, the vehicle can display the current map display in the panoramic map location map; area measurement function, vehicle monitoring system can measure arbitrary polygon area on map; map length measurement function, i.e. vehicle the monitoring subsystem can be measured between any two points of the distance map.

\subsection{The function of the query function module}

The function of vehicle monitoring subsystem can query a location, and can be carried out on the electronic map. The vehicle monitoring subsystem can query the emergency resources in a vehicle. The vehicle monitoring system can display the function of the vehicle, which is a vehicle. The vehicle monitoring system can display the vehicle in the electronic map.

\subsection{The function of the function module of the historical track playback}

Playback functions, namely vehicle monitoring subsystem can play the historical path of the vehicle in the electronic map; back function, namely vehicle monitoring subsystem can play the historical path of the vehicle in the electronic map back when playing; advance feature, namely vehicle monitoring subsystem can play the historical path of the vehicle in the electronic map, can fast forward to speed 
up playback speed; stop function, namely the vehicle monitoring subsystem can stop playing the historical track; a pause function, namely the vehicle monitoring subsystem can temporarily stop the historical track playback; back to the function, namely vehicle surveillance system can real-time playback of historical trajectory. Select the time interval to play the historical trajectory of the vehicle monitoring subsystem can choose which time interval of the historical trajectory of the playback.

\section{Main operating interface of the system}

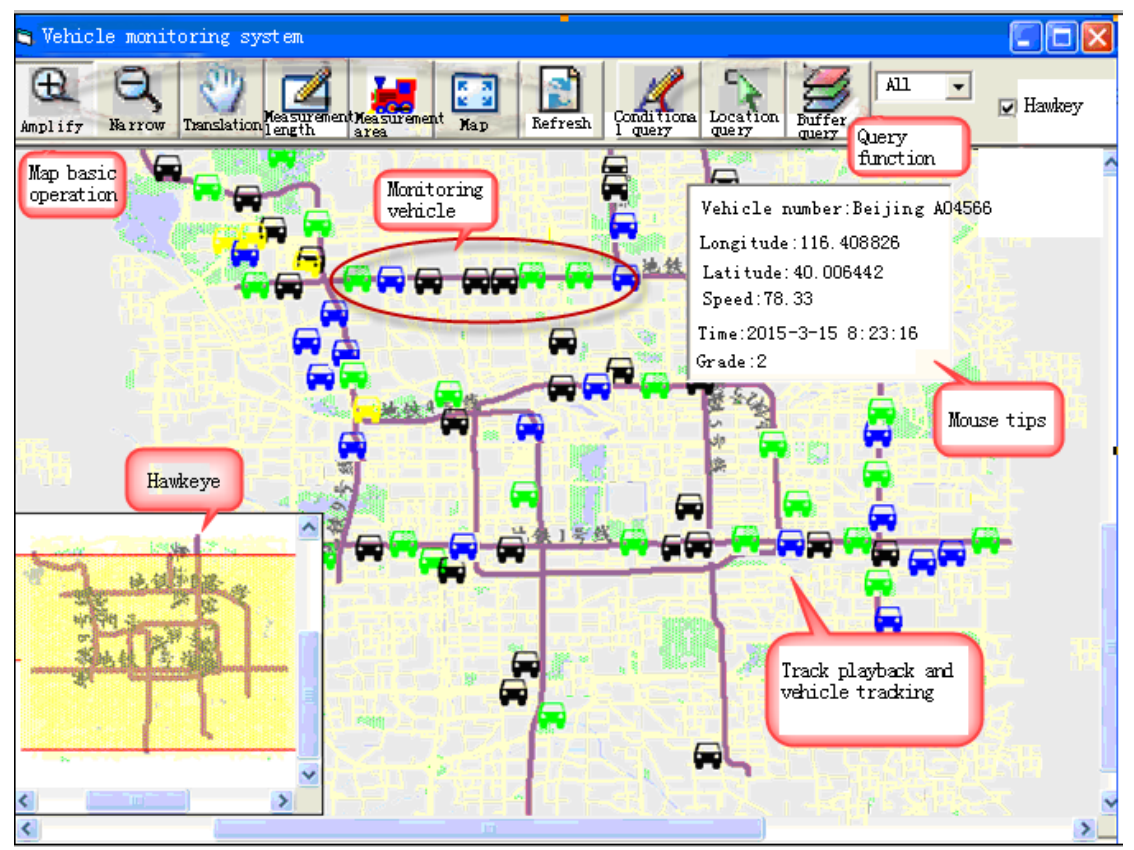

Figure 2. System main interface

As can be seen from Figure 2, you can use the toolbar to complete the operation of the vehicle monitoring system for most of the functions. In the toolbar with map zoom buttons, map reduce button, map button translation, measuring the length of the button, surveying the area button and map button. Map can be completed through the basic operation of the map. We can first click the corresponding button, and then use the mouse on the map for the corresponding operation to complete the corresponding function.

In the toolbar, there are conditions for the query button, the place query button, and the buffer query button. You can complete the query function by these buttons. Condition query: first click the query button, will pop up the vehicle query dialog box, in the dialog box to choose the way of inquiry, is according to the vehicle number query or by vehicle number query. For example, according to the vehicle number query, in the drop-down combo box in the back of the input selection to check the vehicle number, and finally click ok. Location query: first click the location query button, will pop up the location query dialog box, select the location type in the dialog box, and then enter the name or place names in the name or key words, and finally click ok. Buffer query: first click the buffer query button, and then on the map for the corresponding operation, hold down the left mouse button, will show a circle on the map, said the area covered by the mouse, the map will show all of the rescue supplies in this area (hospital).

If you want to play a certain vehicle historical track or to track a vehicle, you can first query the vehicle on the map, and then click the right mouse button, will pop up a shortcut menu, the menu is the main three options: historical track playback, tracking vehicle and tracking. You can select the 
appropriate options in the shortcut menu to complete the corresponding operation, to achieve the function of the historical track playback or tracking vehicle.

\section{Conclusions}

With the great development of urban traffic, the number of vehicles is increasing at an alarming rate. It has brought many problems to traffic management and social security. The vehicle monitoring system provides a very good solution to solve the problem of vehicle management. In this paper, the research of vehicle monitoring system based on global positioning technology, geographic information processing technology, wireless communication technology and computer data processing technology, has developed a global, all-weather, real-time, multi vehicle monitoring system.

\section{References}

1. Zhao Yuexia. Research on the problems and Countermeasures of urban traffic congestion [J]. Shanxi: brand.7(2015)

2. Zhao Zhihui. Research on the status quo and Countermeasures of intelligent transportation in China [J]. Inner Mongolia: Northern Economic.4(2013)

3. Zhang Yan. based on POS and [J]. 7(2015)

4. Bi Juntao. Design of vehicle monitoring system based on Fuzzy vision technology [J]. Beijing: computer measurement and control.9(2014)

5. Ning Heng. Design and implementation of GPS/GPRS/GIS vehicle monitoring system based on [J]. Beijing: micro computer information.8(2010)

6. Song Zhongshan, Bie Lihua, Wang Xin. Analysis of key technology of vehicle monitoring system design [J]. Hubei: Journal of South-Central University For Nationalities (NATURAL SCIENCE EDITION).3(2006)

7. Du Pengcheng. Design and implementation of vehicle monitoring system based on [D]. WebGIS/GPRS Shandong: Shandong University.5(2013) 